\title{
Matching Pursuit and Source Deflation for Sparse EEG/MEG Dipole Moment Estimation
}

\author{
Shun Chi Wu, Member, IEEE, and A. Lee Swindlehurst*, Fellow, IEEE
}

\begin{abstract}
In this paper, we propose novel matching pursuit (MP)-based algorithms for EEG/MEG dipole source localization and parameter estimation for multiple measurement vectors with constant sparsity. The algorithms combine the ideas of MP for sparse signal recovery and source deflation, as employed in estimation via alternating projections. The source-deflated matching pursuit (SDMP) approach mitigates the problem of residual interference inherent in sequential MP-based methods or recursively applied (RAP)-MUSIC. Furthermore, unlike prior methods based on alternating projection, SDMP allows one to efficiently estimate the dipole orientation in addition to its location. Simulations show that the proposed algorithms outperform existing techniques under various conditions, including those with highly correlated sources. Results using real EEG data from auditory experiments are also presented to illustrate the performance of these algorithms.
\end{abstract}

Index Terms-Alternating projection, deflation, dipole source localization, electroencephalography (EEG), matching pursuit (MP), magnetoencephalography (MEG), sparse representations.

\section{INTRODUCTION}

$\mathbf{T}$ HE problem of source localization in electroencephalography (EEG) or magnetoencephalography (MEG) data has attracted significant interest for many years. A large number of localization algorithms have been developed based on models that assume that the sources can be represented as magnetic or electric dipoles with either constant or time-varying magnitude and orientation. The source localization problem involves a high-dimensional inverse problem that in general has an infinite number of solutions. To find a reasonable answer among the set of possible solutions, constraints must be incorporated into the problem. The most commonly used constraint in the literature is the minimum-norm constraint (e.g., [1]), which finds the solution vectors that best match the measurements with the smallest $l_{2}$ residual. The strengths of this approach include low computational cost and smooth reconstructed source spectra. However, $l_{2}$ methods typically produce overly smeared sources that diverge in appearance from the sparse and highly localized nature of brain activity. As a result, researchers have resorted to the use of sparseness constraints in either spatial [2]-[10], spatiotemporal [11], or spatiotemporal frequency [12] domains

Manuscript received June 30, 2012; revised September 20, 2012, December 17, 2012, and March 6, 2013; accepted March 6, 2013. Date of publication March 15, 2013; date of current version July 13, 2013. Asterisk indicates corresponding author

S. C. Wu is with the Department of Electrical Engineering and Computer Science, University of California, Irvine, CA 92697 USA (e-mail: scwu@uci.edu).

*A. L. Swindlehurst is with the Department of Electrical Engineering and Computer Science, University of California, Irvine, CA 92697 USA (e-mail: swindle@uci.edu).

Color versions of one or more of the figures in this paper are available online at http://ieeexplore.ieee.org.

Digital Object Identifier 10.1109/TBME.2013.2253101 to reflect the focal nature of cortical activity. By providing an overcomplete dictionary representing the solution vectors or matrices, a reconstruction problem with the sparseness constraint will search for a solution vector/matrix that not only matches the measurements, but also has as few nonzero entries as possible. This process is also referred to as subspace/basis selection in the literature and typically requires a combinatorial search [5], [13] or a more sophisticated minimization technique (e.g., the expectation-maximization algorithm) [11].

To avoid an exhaustive search for an optimal solution, some low-complexity algorithms have been proposed. Diversity measure minimization techniques initially select all the vectors of the dictionary and eliminate them one by one until a requisite number remains [5], [14]. The FOCal Underdetermined System Solver (FOCUSS) [4] is a famous example belonging to this category, and uses a weighted minimum-norm approach for sequentially reinforcing strong sources and reducing weak ones and ultimately leads to a sparse solution. Algorithms based on other diversity measures (e.g., the $l_{p}$ norm with $p \leq 1$ ) also exist in the literature. Among them, the minimum $l_{1}$-norm constraint is the most popular [2], [3], [6]. Although finding minimum $l_{1}$-norm solutions normally involves a nonlinear minimization procedure, linear programming solutions are possible if the dipole orientation at each grid point is somehow already known (e.g., estimated using the minimum $l_{2}$-norm approach in advance) [6]-[8]. However, if the predefined orientations deviate from the true orientations, estimation errors are inevitable. Ad hoc methods for dealing with the need for knowledge of the dipole orientation have been proposed; for example, a modified minimum $l_{1}$-norm approach is adopted for orientation estimation in [8], and in [7] only the sign of the orientation component at each grid point is used in the $l_{1}$-norm approach instead of the actual orientation, but in both cases information regarding the orientation is still required.

As an alternative to direct minimization of the $l_{1}$ norm, matching pursuit (MP) search techniques [14]-[16] have been proposed that sequentially add vectors from the dictionary matrix into the set for representing the measurements and then remove their contribution from the measurements before searching for additional sources. These approaches significantly reduce the search effort since the original multibasis-vector selection problem is replaced with several smaller problems where only a single entry from the dictionary matrix that best matches the residual measurements is sought. In addition, they do not require prior information about the dipole orientations in order to obtain source location estimates.

An important distinction arises with EEG/MEG localization problems compared to the standard modeling assumptions made 
for MP-based methods, since the vector signal produced by a given dipole source is a linear combination of lead field vectors in either two or three (EEG) dimensions, corresponding to the dipole orientation. (Only two dimensions are required for MEG data since the radial component of the magnetic dipole generates no external magnetic fields [17].) When projecting out the contribution of a given source, one is faced with the problem of either projecting away the space spanned by the component lead field vectors at the given location, or estimating the dipole moment and projecting away only the 1-D space corresponding to the linear combination. The former approach is easier to implement, but leads to a performance degradation since signal dimensions beyond those that are necessary are projected away. The so-called RAP-MUSIC algorithm [18] is an example of an MP-like algorithm that uses the latter approach with dipole moment estimation. The computational advantage of not estimating the dipole moment is greatest for models with multiple measurement vectors (MMVs) [16], [19] when the dipole orientation is time-varying or arbitrary at each time instant [4]. We will refer to this as the "unstructured" dipole model, while the term "structured" dipole model will refer to the case where the orientation is constant with time [17], [20], [21]. Both models/approaches will be considered later.

In this paper, we consider a refinement to MP-based methods for models based on MMVs with constant sparsity structure. The new approach, referred to as source-deflated matching pursuit (SDMP), iteratively refines a set of source location estimates by reestimating the position of each source after projecting away the contributions from all other estimated sources. Stated another way, unlike MP methods that stop once one has extracted the desired number of sources, SDMP continues to refine the position estimates (and the dipole moment estimates for structured models) by removing the contributions from all sources except the current one of interest. The advantage of such an approach is that it tends to eliminate the interference from residual sources present in each step of the MP methods. Furthermore, this approach allows insertions and deletions of dictionary vectors at each step in order to ensure a better recovery performance, since an incorrect selection early in the iterations (e.g., an inaccurate initial index set) can be neutralized once it is removed from the index set. Techniques have been proposed in the compressive sensing literature that perform such deletions and insertions [22]-[24], and while they have not been explicitly applied to problems where multiple dictionary vectors are associated with each source (as in the EEG/MEG problem), generalizations are not hard to derive. We will compare our source deflation (SD) approach with one such generalization in our simulations.

The SD concept is similar to the alternating projection (AP) algorithm [25]. One key difference is that AP uses a continuous parameter search while SDMP searches for the appropriate index of the overcomplete dictionary. The advantage of the SDMP approach is that it prevents residual energy from a previously detected strong source from leaking into subsequent steps and causing spurious additional sources to be detected near it. We will also show how SDMP can be used to estimate the dipole orientation in addition to its location. The AP-based method pro- posed for diversely polarized arrays (similar to the EEG/MEG model) cannot take advantage of the structured model in estimating the orientation vector [26]. SD has been previously applied to source localization for MEG for the unstructured case in [27]. Our work differs in that we focus on estimating the dipole orientation assuming the structured signal model. In particular, we present SDMP approaches that use MUltiple SIgnal Classification (MUSIC) [17], [28] so that the estimation of the source position and its orientation can be decoupled and estimated efficiently.

The remainder of the paper is outlined as follows. In the next section, we present the data model and assumptions made in this study. Section III discusses the application of MP algorithms to multidimensional source localization for the unstructured model. Estimation for the structured model is then considered in Section IV. Simulation and experimental examples and results are described and discussed in Sections V and VI, respectively. Finally, some conclusions are offered in Section VII. An earlier version of the results presented in this paper appeared in [10].

\section{DATA MODEL AND ASSUMPTIONS}

\section{A. Data Model}

Although from a mathematical standpoint, the techniques we develop are applicable to both EEG and MEG source localizations, we develop the model here only for the EEG case. In the standard data model for EEG source localization, the cortex or brain region of interest is divided into $n$ small disjoint candidate locations, and activated locations are modeled as equivalent current dipole sources [29], [30]. The signal received at an $m$ element electrode array at time $t$ due to the $k$ th source at the 3-D location/cell $\mathbf{r}_{k}$ is given by $\mathbf{G}\left(\mathbf{r}_{k}\right) \mathbf{m}_{k}(t)$, where $\mathbf{m}_{k}(t) \in \mathbb{R}^{3 \times 1}$ is the dipole moment and

$$
\mathbf{G}\left(\mathbf{r}_{k}\right)=\left[\mathbf{g}_{x}\left(\mathbf{r}_{k}\right) \mathbf{g}_{y}\left(\mathbf{r}_{k}\right) \mathbf{g}_{z}\left(\mathbf{r}_{k}\right)\right] \in \mathbb{R}^{m \times 3}
$$

is the lead field matrix (LFM) with $\mathbf{g}_{x}\left(\mathbf{r}_{k}\right), \mathbf{g}_{y}\left(\mathbf{r}_{k}\right)$, and $\mathbf{g}_{z}\left(\mathbf{r}_{k}\right)$ representing the $m \times 1$ lead field vectors corresponding to unit amplitude sources at $\mathbf{r}_{k}$ and oriented in the $x-, y$-, and $z$-directions, respectively. In practice, these vectors can be derived from multilayer spherical head models [21], [30] or based on realistic head measurements taken from computed tomography or magnetic resonance imaging data [31]. If the dipole orientation is time invariant, then $\mathbf{m}_{k}(t)=\phi_{k} s_{k}(t)$, where $s_{k}(t) \in \mathbb{R}$ and $\phi_{k} \in \mathbb{R}^{3 \times 1}$ represent, respectively, the dipole moment magnitude and orientation for source $k$. The dipole orientation is normalized to have unit length: $\left\|\phi_{k}\right\|=1$.

Assuming that there are $n$ possible source locations/cells in the cortical region of interest, the total array output is modeled as a linear superposition of the contributions from these cells plus noise

$$
\mathbf{x}(t)=\mathbf{G m}(t)+\mathbf{n}(t)
$$

where $\mathbf{G}=\left[\begin{array}{lll}\mathbf{G}\left(\mathbf{r}_{1}\right) & \cdots & \mathbf{G}\left(\mathbf{r}_{n}\right)\end{array}\right] \in \mathbb{R}^{m \times 3 n}, \mathbf{m}(t)=\left[\mathbf{m}_{1}^{T}(t)\right.$ $\left.\cdots \mathbf{m}_{n}^{T}(t)\right]^{T} \in \mathbb{R}^{3 n \times 1}$, and $\mathbf{n}(t)$ represents noise and interference. Due to the sparse and highly localized nature 
of the brain activity, most components of the moment vector $\mathbf{m}(t) \in \mathbb{R}^{3 n \times 1}$ are zero.

In most applications, MMVs are taken. Assuming $N_{t}$ such measurements, and that the source locations are constant during the measurements (constant sparsity structure), the MMV model can be succinctly written as

$$
\begin{aligned}
\mathbf{X} & =\mathbf{G M}+\mathbf{N} \\
& =\left[\begin{array}{lll}
\mathbf{G}\left(\mathbf{r}_{1}\right) & \cdots & \mathbf{G}\left(\mathbf{r}_{n}\right)
\end{array}\right]\left[\begin{array}{c}
\mathbf{M}_{1} \\
\vdots \\
\mathbf{M}_{n}
\end{array}\right]+\mathbf{N}
\end{aligned}
$$

where $\mathbf{M}_{k} \in \mathbb{R}^{3 \times N_{t}}$ corresponds to the moment of the dipole located at $\mathbf{r}_{k}, \mathbf{X}=\left[\mathbf{x}(1), \ldots, \mathbf{x}\left(N_{t}\right)\right]$, and $\mathbf{N}$ is defined similarly. In the structured dipole model, $\mathbf{M}_{k}$ is rank one

$$
\mathbf{M}_{k}=\phi_{k} \mathbf{s}_{k}
$$

where $\mathbf{s}_{k}=\left[s_{k}(1) \cdots s_{k}\left(N_{t}\right)\right]$.

\section{UNSTRUCTURED MODEL: MP AND BIAS FACTORS}

The highly localized nature of evoked brain activity results in highly sparse moment vectors once an overcomplete dictionary matrix is adopted. This means that only a small number of columns of the dictionary matrix $\mathbf{G}$ will have an effect on the measurements. In general, optimal selection of the lead field matrices that best represent the measurements requires a combinatorial search. However, the cost of such a search is prohibitive even for a moderate number of sources [5]. MP search methods can substantially reduce the computational effort by sequentially rather than simultaneously selecting vectors in $\mathbf{G}$ to include in the set for representing the measurement matrix, projecting out the contribution of each source as it is identified.

A modification to standard MP algorithms is necessary for the multidimensional EEG model, since in general each source results in three rather than one nonzero entries in $\mathbf{m}(t)$. If an MP method locates a source at $\mathbf{r}_{k}$, without knowledge of the corresponding entries $\mathbf{m}_{k}(t)$ or an attempt to estimate it (which is difficult during the MP iterations), the projection step must eliminate the entire 3-D subspace corresponding to $\mathbf{G}\left(\mathbf{r}_{k}\right)$ rather than just the actual 1-D contribution $\mathbf{G}\left(\mathbf{r}_{k}\right) \mathbf{m}_{k}(t)$. In the following two sections, we show how to modify the two most common MP-based methods to take into account the fact that each source corresponds to multiple dictionary vectors. We also discuss the residual interference factor that can lead to bias in the resulting location estimates. We then present the SDMP approach for unstructured sources at the end of the section.

\section{A. Basic Matching Pursuit (BMP)}

The BMP search algorithm [15], [16] chooses a new source location at each step by finding the index of the grid point whose dictionary vector best matches the residual measurements. To generalize to the EEG case where a source at location $\mathbf{r}$ produces signal components in the 3-D space spanned by the LFM G $(\mathbf{r})$, we choose index $k_{p}$ as the $p$ th source if its LFM is most closely aligned with the residual measurements $\mathbf{X}_{p-1}$, as follows:

$$
\begin{aligned}
& k_{p}=\arg \max _{k}\left\|\mathbf{P}_{\mathbf{G}_{k}} \mathbf{X}_{p-1}\right\|_{F}^{2} \\
& \text { for } k \in\{1,2, \ldots, n\}
\end{aligned}
$$

where $I_{p-1}$ is the index set for the first $p-1$ sources and $\mathbf{P}_{\mathbf{G}_{k}}=$ $\mathbf{G}\left(\mathbf{r}_{k}\right)\left[\mathbf{G}^{T}\left(\mathbf{r}_{k}\right) \mathbf{G}\left(\mathbf{r}_{k}\right)\right]^{-1} \mathbf{G}^{T}\left(\mathbf{r}_{k}\right)$. The index set and residual measurement matrix $\mathbf{X}_{p-1}$ are updated as follows:

$$
\begin{aligned}
I_{p} & =I_{p-1} \cup\left\{k_{p}\right\} \\
\mathbf{X}_{p} & =\left(\mathbf{I}-\mathbf{P}_{\mathbf{G}_{k_{p}}}\right) \mathbf{X}_{p-1} .
\end{aligned}
$$

The process repeats until a certain stopping criterion is reached, for example when the residual becomes sufficiently small or a solution with the desired level of sparsity is obtained.

\section{B. Order Recursive Matching Pursuit (ORMP)}

The main difference between ORMP and BMP is that not only the index set and the residual measurement matrix but also the dictionary matrix is modified at each step of the algorithm [15], [16]. ORMP is similar in spirit to the Orthogonal MP algorithm of [32]. To generalize to the EEG data model, we choose the candidate location corresponding to index $k_{p}$ as the $p$ th source if its "filtered" LFM best aligns with the residual measurement matrix $\mathbf{X}_{p-1}$ :

$$
\begin{gathered}
k_{p}=\arg \max _{k}\left\|\mathbf{P}_{\mathbf{G}_{k}^{(p-1)}} \mathbf{X}_{p-1}\right\|_{F}^{2} \\
\text { for } k \in\{1,2, \ldots, n\} \backslash I_{p-1}
\end{gathered}
$$

where $\mathbf{P}_{\mathbf{G}_{k}^{(p-1)}}=\mathbf{G}_{k}^{(p-1)}\left[\mathbf{G}_{k}^{(p-1) T} \mathbf{G}_{k}^{(p-1)}\right]^{-1} \mathbf{G}_{k}^{(p-1) T}$ and we have used the simplified notation $\mathbf{G}_{k}^{(p-1)}=\mathbf{G}^{(p-1)}\left(\mathbf{r}_{k}\right)$. The updates to the index set, residual measurement matrix, and the dictionary are given by

$$
\begin{aligned}
I_{p} & =I_{p-1} \cup\left\{k_{p}\right\} \\
\mathbf{X}_{p} & =\left(\mathbf{I}-\mathbf{P}_{\mathbf{G}_{k p}^{(p-1)}}\right) \mathbf{X}_{p-1} \\
\mathbf{G}_{k}^{(p)} & =\left(\mathbf{I}-\mathbf{P}_{\mathbf{G}_{k_{p}}^{(p-1)}}\right) \mathbf{G}_{k}^{(p-1)} .
\end{aligned}
$$

The update to the dictionary is added because when we update the residual measurement matrix by projecting it onto the subspace orthogonal to $\mathbf{G}_{k_{p}}^{(p-1)}$, not only will the contribution of the LFM $\mathbf{G}_{k_{p}}^{(p-1)}$ to $\mathbf{X}_{p-1}$ be removed, but the LFMs of all residual sources will be altered as well. In this way, ORMP effectively prevents the energy from "leaking" back into subspaces spanned by previously selected LFMs as in BMP.

\section{Residual Interference in MP Estimators}

A key factor that biases the EEG position estimates when using MP algorithms such as BMP and ORMP for source localization is due to residual interference when more than one source is present [33]. Assuming $N_{S}$ total sources, the spectrum generated by the BMP or ORMP cost function when finding the $p$ th source will ideally have a single global maximum, and the index corresponding to the global maximum will correspond to the location of one of the sources. However, because of the 
"spatial interference" among different sources, the formation of the global maximum is not solely due to the LFM of some specific candidate region; instead, it might be a "compromise" between all $N_{S}-p$ residual sources, which will lead to biased estimates. For example, if two sources are so close together as to be normally unresolvable, the maximum of the cost function may occur at some point between the two sources. Even though a subsequent MP step will find the second source, the estimate of the first source has been biased due to the presence of the other. For EEG applications, the problem manifests itself not only for closelyspaced sources, but also when weak deep-brain sources are present [4].

\section{Source Deflated Matching Pursuit}

The idea behind the SD is the observation that the problem of interference from residual sources is obviously eliminated when only one source is present. If the original multisource search can be replaced by a series of (nearly) unbiased singlesource searches, the influence of the residual-source interference on localization accuracy can be largely eliminated. As such, the SDMP approach iteratively refines a set of source location estimates (obtained using, for example BMP or ORMP) by reestimating the position of each source after projecting away the contributions from all other estimated sources. Instead of stopping once the desired number of sources has been extracted, SDMP continues to refine the obtained position estimates by removing at each step the contributions from all sources but one, and then alternating through all of the sources one at a time. A detailed description of the SDMP approach for the unstructured model is outlined as follows.

Initialization Stage: Obtain an initial index set of $N_{S}$ source locations (e.g., using BMP or ORMP)

$$
I_{0}=\left\{k_{1}^{0}, k_{2}^{0}, \ldots, k_{N_{S}}^{0}\right\} .
$$

Deflation Stage: Given the MMV matrix X, let $N_{\max }$ represent the maximum number of iterations used to refine each location estimate.

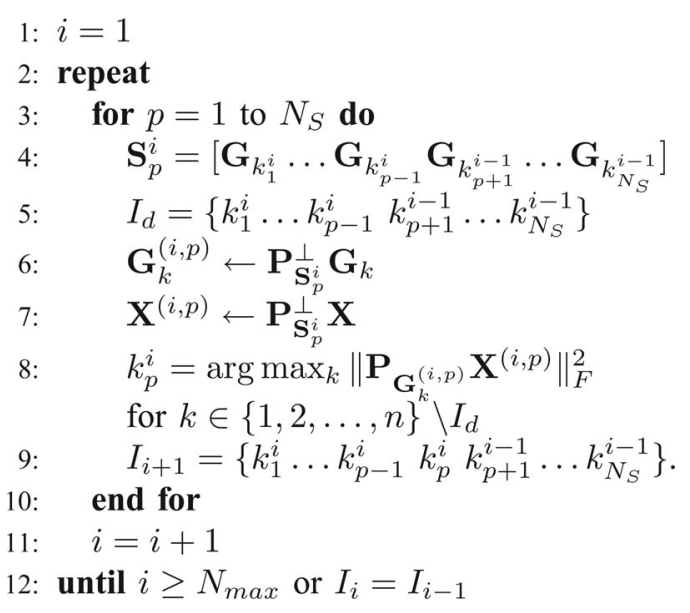

The projection matrix $\mathbf{P}_{\mathbf{S}_{p}^{i}}^{\perp}=\mathbf{I}-\mathbf{S}_{p}^{i}\left[\left(\mathbf{S}_{p}^{i}\right)^{T} \mathbf{S}_{p}^{i}\right]^{-1}\left(\mathbf{S}_{p}^{i}\right)^{T}$ is used to suppress the contribution of the sources at locations other than the one for which a fine search is being conducted.
As mentioned earlier, the difference between SDMP and the AP algorithm of [26] comes in line 8, where a search over the dictionary index is used instead of a continuous parameter estimation. Our simulations indicate that a relatively small number of iterations is required before the location indices are no longer updated by the SDMP iterations.

\section{MP FOR the StRUCtUREd MOMENT MOdEL}

Line 8 of the aforementioned SDMP algorithm implements an unstructured maximum likelihood (ML) estimation for the 3-D source location similar to that in [34]. Again, we refer to this as an unstructured estimator since the dipole orientation can be arbitrary at every time instant $t$. However, this assumption will lead to degraded estimation performance when the underlying EEG/MEG activity is governed by a structured or time-invariant dipole moment. Although one could modify line 8 to accommodate a structured model, the location and orientation parameters are not separable in the resulting ML criterion. In this way, the dimension of the search space would increase dramatically since instead of just $n$ cells to search, one would have to expand the size of the search to $n \times n_{o}$ "cells," where $n_{o}$ represents the number of discretized candidate orientation vectors possible at each source location.

In this section, we present an alternative to the ML estimation step in line 8 of the SDMP algorithm for structured source models. The alternative is based on MUSIC [17], [28] which allows for continuous-valued estimates of the dipole orientation to be obtained separately from the search for the source index. Similarly, one can easily come up with another alternative based on linearly constrained minimum variance beamforming [35]. Before presenting the algorithms, we define the covariance of the residual measurements and its eigendecomposition as follows:

$$
\begin{aligned}
\hat{\mathbf{R}}_{r}^{(i, p)} & =\frac{1}{N_{t}} \mathbf{X}^{(i, p)} \mathbf{X}^{(i, p) T}=\sum_{i=1}^{m} \lambda_{i} \mathbf{e}_{i}^{(i, p)} \mathbf{e}_{i}^{(i, p) T} \\
& =\lambda_{1} \mathbf{e}_{1}^{(i, p)} \mathbf{e}_{1}^{(i, p) T}+\mathbf{E}_{N}^{(i, p)} \boldsymbol{\Lambda}_{N} \mathbf{E}_{N}^{(i, p) T}
\end{aligned}
$$

where the eigenvalues are ordered as $\lambda_{1} \geq \cdots \geq \lambda_{m}$, the vector $\mathbf{e}_{1}$ is the eigenvector corresponding to the largest eigenvalue $\lambda_{1}$, and $\mathbf{E}_{N}=\left[\mathbf{e}_{2}, \ldots, \mathbf{e}_{m}\right]$ contain the remaining eigenvectors. We refer to $\mathbf{e}_{1}$ and $\mathbf{E}_{N}$ as the signal and noise subspace eigenvectors, respectively. Since there is ideally only one source left in the SD residual measurements, the signal subspace at line 8 of the SDMP algorithm is 1-D.

\section{A. Source Deflated MUSIC (SDMUSIC)}

Under the SDMP framework, MUSIC [17], [21], [28] selects the source index $k_{p}^{i}$ as the one whose filtered LFM, $\mathbf{G}_{k}^{(i, p)} \phi_{p}$, is most orthogonal to the noise subspace $\mathbf{E}_{N}^{(i, p)}$ :

$$
\begin{aligned}
k_{p}^{i}, \hat{\boldsymbol{\phi}}_{p} & =\arg \max _{k, \boldsymbol{\phi}_{p}} V_{\mathrm{MU}}\left(k, \boldsymbol{\phi}_{p}\right) \\
& =\arg \max _{k, \boldsymbol{\phi}_{p}} \frac{\boldsymbol{\phi}_{p}^{T} \mathbf{G}_{k}^{(i, p) T} \mathbf{G}_{k}^{(i, p)} \boldsymbol{\phi}_{p}}{\boldsymbol{\phi}_{p}^{T} \mathbf{G}_{k}^{(i, p) T} \mathbf{E}_{N}^{(i, p)} \mathbf{E}_{N}^{(i, p) T} \mathbf{G}_{k}^{(i, p)} \boldsymbol{\phi}_{p}}
\end{aligned}
$$


where $\phi_{p}$ is the orientation of the $p$ th dipole source. At first glance, it appears that, like the ML algorithm, maximizing (13) will also require the orientation space to be partitioned. However, unlike ML, the form of (13) allows one to decouple the estimation of the source location and its orientation, and thus leads to an efficient solution.

If $\phi_{p}$ is constrained to be unit norm (i.e., $\phi_{p}^{T} \phi_{p}=1$ ), then maximizing the MUSIC criterion is equivalent to finding the solution of the following generalized eigenvalue problem:

$$
\begin{gathered}
k_{p}^{i}=\arg \max _{k} \lambda_{\max }(k) \quad \text { such that } \\
\mathbf{G}_{k}^{(i, p) T} \mathbf{G}_{k}^{(i, p)} \hat{\boldsymbol{\phi}}_{p}=\lambda_{\max }\left(k_{p}^{i}\right) \mathbf{G}_{k}^{(i, p) T} \mathbf{E}_{N}^{(i, p)} \mathbf{E}_{N}^{(i, p) T} \mathbf{G}_{k}^{(i, p)} \hat{\boldsymbol{\phi}}_{p}
\end{gathered}
$$

where $\lambda_{\max }(\cdot)$ represents the largest of the $m$ nonnegative generalized eigenvalues. The source index is selected by viewing $\lambda_{\max }(k)$ as a function of the index $k$ and then searching for its own maximum. The orientation of the dipole for the selected region $k_{p}^{i}$ is then taken to be the unit-norm eigenvector associated with $\lambda_{\max }\left(k_{p}^{i}\right)$. The SDMUSIC algorithm is obtained by replacing line 8 with (14).

Note that the SD approach immunizes SDMUSIC from the poor performance of MUSIC-based algorithms that occurs with highly correlated sources. Provided that the initial estimates are obtained using a method insensitive to high correlation (e.g., ML-based methods such as BMP or ORMP), the SD step removes the influence of any sources correlated with the one of interest. We will illustrate the robustness of SDMUSIC to highly correlated sources in the simulation examples of the following section.

\section{B. Orientation Estimation for Other MP-Based Algorithms}

While the unstructured MP algorithms of Section III do not find the dipole moments as part of their implementation, we show here that an estimate of the structured dipole orientation can still be obtained once all the location estimates are found. To see this, let $\mathbf{r}_{1}^{S}, \ldots, \mathbf{r}_{N_{S}}^{S}$ represent the estimated source indices, and suppose these source locations accurately represent the observations obtained in the model of (2) and (3), so that

$$
\begin{aligned}
& \mathbf{X}=\left[\begin{array}{lll}
\mathbf{G}\left(\mathbf{r}_{1}^{S}\right) \cdots & \mathbf{G}\left(\mathbf{r}_{N_{S}}^{S}\right)
\end{array}\right]\left[\begin{array}{c}
\mathbf{M}_{1}^{S} \\
\vdots \\
\mathbf{M}_{N_{S}}^{S}
\end{array}\right]+\mathbf{N} \\
& =\mathbf{G}_{S} \mathbf{M}_{S}+\mathbf{N}
\end{aligned}
$$

where $\mathbf{M}_{k}^{S} \in \mathbb{R}^{3 \times N_{t}}$ is the moment of the dipole located at $\mathbf{r}_{k}^{S}$ and $\mathbf{G}_{S}$ is the collection of LFMs associated with the estimated source locations, and where we have dropped the terms associated with inactive source cells. An unstructured estimate of the moment matrix $\mathbf{M}_{S}$ can be obtained via

$$
\hat{\mathbf{M}}_{S}=\left(\mathbf{G}_{S}^{T} \mathbf{G}_{S}\right)^{-1} \mathbf{G}_{S}^{T} \mathbf{X}
$$

While the dipole orientations are not directly revealed in (16), they can be determined from $\hat{\mathbf{M}}_{S}$ in a straightforward way. The $3 N_{S} \times N_{t}$ matrix $\hat{\mathbf{M}}_{S}$ can be partitioned into $N_{S}$ blocks of dimension $3 \times N_{t}$ that represent estimates of the moment for each individual source. Ideally, these blocks, denoted as $\hat{\mathbf{M}}_{k}^{S}$, should be rank one and satisfy $\hat{\mathbf{M}}_{k}^{S}=\phi_{k}^{S} \mathbf{s}_{k}^{S}$. Since the product $\phi_{k}^{S} \mathbf{s}_{k}^{S}$ is rank one, a straightforward solution to the problem under the constraint $\left\|\phi_{k}^{S}\right\|=1$ is to set the estimate $\hat{\phi}_{k}$ equal to the left singular vector of $\hat{\mathbf{M}}_{k}^{S}$ with the largest singular value. In the next section, we will compare the accuracy of estimates obtained via this two-stage process with those found by SDMUSIC.

\section{Simulation EXAmPLeS}

EEG simulations involving 61 electrodes from the 10-10 system [36] were conducted. The LFMs were calculated using the approximate method of [30], in which the head was modeled as a multilayer sphere with radii and conductivities specified in [37]. The brain surface was modeled as a spherical shell and partitioned into 1279 uniformly distributed candidate source cells separated by $0.5 \mathrm{~cm} .{ }^{1}$ Thus, each source was assumed to lie in one of these cells on the spherical brain surface. The structured dipole model was used. The moment magnitude waveforms $s_{k}(t)$ were chosen to be typical averaged evoked response potentials (ERPs) as in [38] and all source ERPs were assumed to be uncorrelated (except for one example noted later) and have equal power. Spatially and temporally white noise was added to the observed data, and the strength of the noise was specified by a given SNR, defined as $\|\mathbf{G M}\|_{F}^{2} /\|\mathbf{N}\|_{F}^{2}$. The spatial white noise model is based on the assumption that any spatially correlated interference has already been suppressed by either a prewhitening or projection step prior to the estimation [20], [21].

For a given source position and orientation $\mathbf{r}^{S}$ and $\phi^{S}$, and an estimated position and orientation $\mathbf{r}^{E}$ and $\phi^{E}$, we claim a "success" for a given algorithm if $\left\|\mathbf{r}^{S}-\mathbf{r}^{E}\right\| \leq d_{\mathrm{th}}$, and $\angle\left(\phi^{S}, \phi^{E}\right) \leq o_{\text {th }}$, where $d_{\text {th }}$ and $o_{\text {th }}$ are the threshold distance and angle metrics, respectively. The RMS position and orientation estimation error for a given source are defined as

$$
\begin{aligned}
\operatorname{Loc}_{\mathrm{Err}} & =\sqrt{\frac{1}{N_{\text {succ }}} \sum_{i=1}^{N_{\text {succ }}}\left\|\mathbf{r}_{i}^{S}-\mathbf{r}_{i}^{E}\right\|^{2}} \\
\text { Orn }_{\text {Err }} & =\sqrt{\frac{1}{N_{\text {succ }}} \sum_{i=1}^{N_{\text {succ }}}\left[\angle\left(\phi_{i}^{S}, \phi_{i}^{E}\right)\right]^{2}}
\end{aligned}
$$

where $N_{\text {succ }}$ denotes the number of estimates that were deemed a "success" for that algorithm. The RMS errors plotted in the simulation figures are averages of $\mathrm{Loc}_{\mathrm{Err}}$ and $\mathrm{Orn}_{\mathrm{Err}}$ taken over all of the sources. The "success rate" of a given algorithm is the percentage of trials for which both the location and orientation estimates obtained by the algorithm fell within $d_{\mathrm{th}} \mathrm{cm}$ and $o_{\mathrm{th}}$ radians of the true values, respectively. The RMS errors indicate the estimation accuracy achieved when the algorithms satisfy the criterion for success; outliers are removed so that the resulting RMS values will be meaningful. We will see that the proposed

\footnotetext{
${ }^{1}$ All distance measurements given in this section are "straight line" or Euclidean distances.
} 
SD methods not only have significantly fewer outliers; their RMS estimation errors are considerably lower even though the competing algorithms have many more outliers removed from their RMS calculation. For all of the simulations, we assumed $d_{\mathrm{th}}=1 \mathrm{~cm}$ and $o_{\mathrm{th}}=0.5 \mathrm{rad}$. Other reasonable choices for $d_{\mathrm{th}}$ and $o_{\text {th }}$ yield similar results.

In the examples presented later, we study the impact of various factors on the performance of the proposed algorithms. In addition, for the purpose of comparison, we also include results obtained by the vector-based minimum $l_{1}$-norm (VESTAL) solution of [7], the minimum current estimates (MCE) approach of [6], and the single-best replacement (SBR) algorithm in [24] adapted for EEG models with multiple dictionary vectors per source. Of these three algorithms, the SBR approach is emphasized since it yields the best performance. In the generalization of SBR, sources are iteratively added or removed one at a time, and a weighted 2-norm plus 0-norm criterion is used to determine which addition or deletion (if any) is maintained for the next sweep through the source locations. A difficulty with this approach is the choice of the $a d$ hoc weighting factor; in our simulations, we experimented with several weightings to find the one that gave the best performance.

\section{A. Single Trial Example}

In the first example, we present representative results for a single trial with six sources with an SNR of $5 \mathrm{~dB}$ for each source. Results are given in Fig. 1, with the magenta dots representing the true locations of the sources. Note that the label "SDMU" in the figure refers to SDMUSIC. While all of the algorithms reconstruct sparse and focal activity around the true source positions, MUSIC and BMP are able to localize only a subset of the sources. MCE and VESTAL show some spurious activity as well, which is likely due to errors in the preestimated orientations. SD and SBR perform similarly in this example, accurately locating all of the sources without any false estimates.

\section{B. Impact of Number of Sources}

To show performance averaged over multiple trials, an experiment was conducted where in each trial, sources with random orientations were quasi-randomly placed on the brain surface. The source locations were constrained to be separated by at least $5 \mathrm{~cm}$. The SNR was set at $5 \mathrm{~dB}$ and 100 trials were conducted for each case involving different numbers of sources from 2 to 10 (all algorithms perform identically for one source). The estimation errors, success rate, and computational load (measured in seconds of MATLAB computation) required per trial are summarized in Fig. 2. We see from the figure that when more than one source is present, the effect of residual interference degrades the performance of BMP and ORMP, and the degradation increases as more sources are added. The average SBR performance is similar to ORMP, while unstructured SDMP and structured SDMUSIC achieve considerably better success rates as well as RMS position and orientation estimation errors under all conditions.

Since SDMP normally takes two to four iterations through all of the sources in order to converge, the computational load
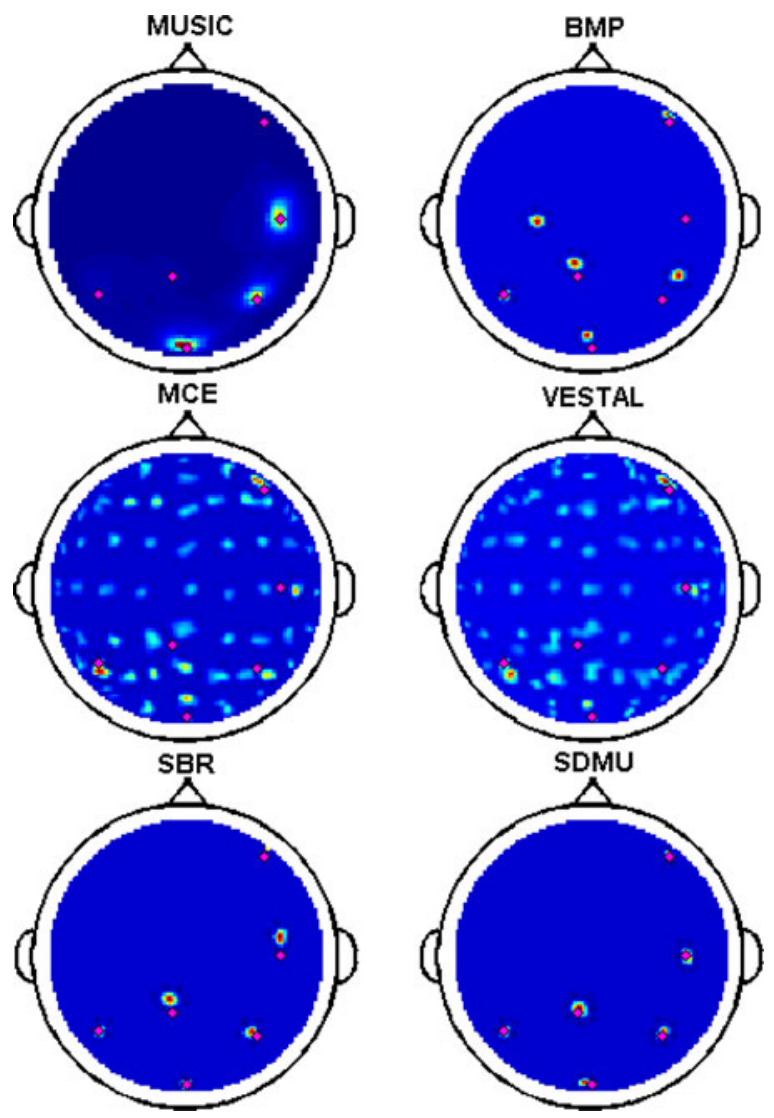

Fig. 1. Estimated source activity obtained by various algorithms for a single trial with $5 \mathrm{~dB}$ SNR. Magenta dots represent the true positions of the sources of interest. (a) Average Time Used Per Tria

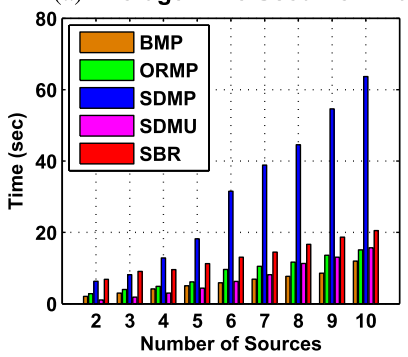

(c) Position

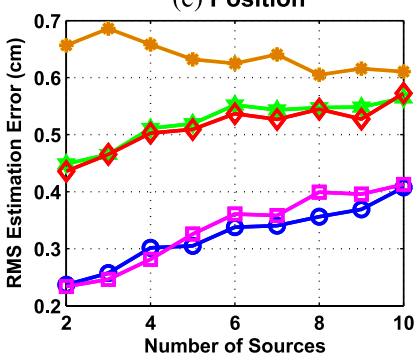

(b) Success Rate

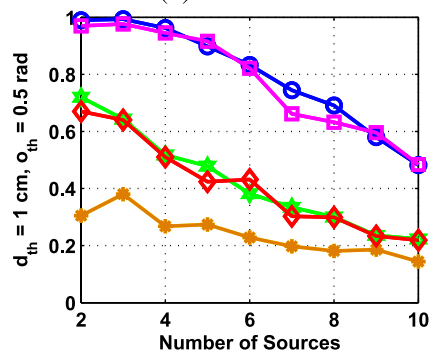

(d) Orientation

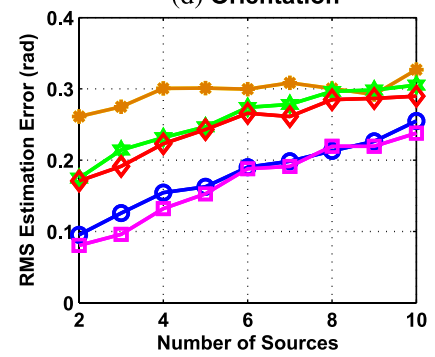

Fig. 2. Influence of the number of sources on performance. (a) Average MATLAB computation time required per trial, (b) success rate, (c) RMS position estimation error, and (d) RMS orientation estimation error. $\mathrm{SNR}=5 \mathrm{~dB}$, minimum source separation distance $=5 \mathrm{~cm}, d_{\mathrm{th}}=1 \mathrm{~cm}$ and $o_{\mathrm{th}}=0.5 \mathrm{rad}$. 

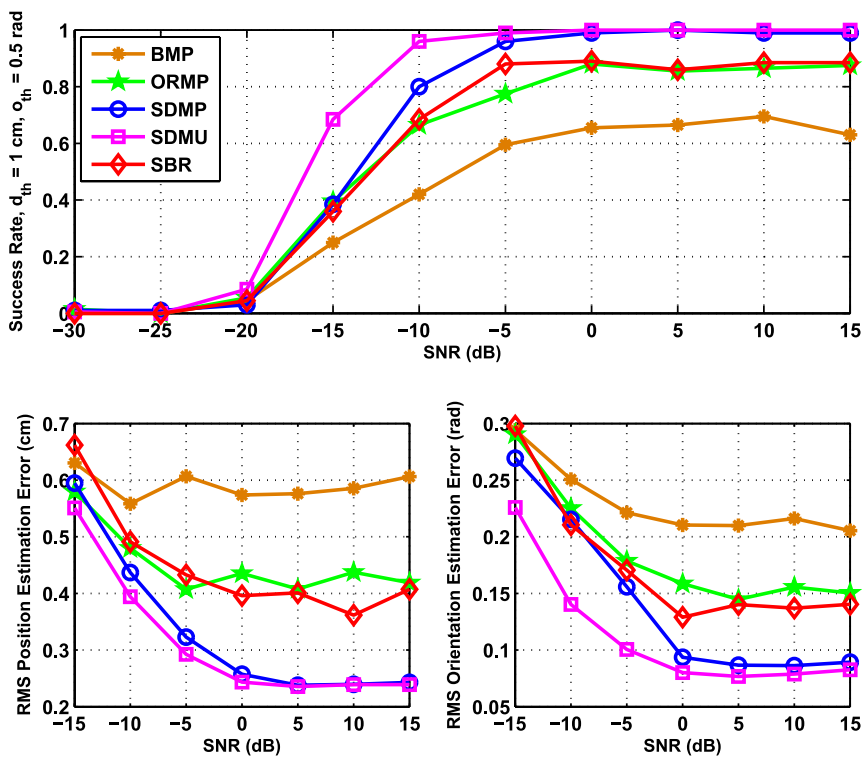

Fig. 3. Impact of SNR on performance. Top: success rate, bottom left: RMS position estimation error, bottom right: RMS orientation estimation error as a function of SNR with source separation distance $=7.5 \mathrm{~cm}, d_{\mathrm{th}}=1 \mathrm{~cm}$ and $o_{\text {th }}=0.5 \mathrm{rad}$.

is naturally two to four times that of BMP or ORMP. BMP, ORMP, and SBR are in turn more computationally expensive than SDMUSIC since each index update requires calculation of a relatively large projection matrix for nearly all candidate source locations. By contrast, SDMUSIC requires computation of $\mathbf{E}_{N}^{(i, p)}$ only once. This more than offsets the fact that SDMUSIC iterates through all the sources multiple times.

\section{Impact of $S N R$}

A pair of sources with random orientations was randomly placed on the brain surface with a fixed separation of $7.5 \mathrm{~cm}$. The SNR was varied from -15 to $15 \mathrm{~dB}$ and 100 trials were conducted for each SNR value. Fig. 3 shows the results. As in the previous case, SDMUSIC achieves superior results due to its ability to fully exploit the time-invariant dipole orientation model.

Correlated cortical activities often occur in practice and are thought to be the result of some type of functional connectivity between different regions in the brain [39], [40]. More heuristically, we may expect to see highly correlated sources in EEG signals at different locations that are the result of the same stimulus; these signals are excited simultaneously and often have similar evoked responses. In the next simulation, we study the effect of temporal correlation on the performance of the proposed algorithms. A pair of sources separated by $7.5 \mathrm{~cm}$ and with random orientations was randomly placed on the brain surface, and the source ERPs were generated to be $95 \%$ correlated with each other. Fig. 4 shows the results when the SNR is varied from -15 to $15 \mathrm{~dB}$, with 100 trials conducted at each SNR. Comparing Fig. 4 with results from the uncorrelated case in Fig. 3, we see that all algorithms suffer some performance loss with highly correlated signals, but the SD-based approaches are
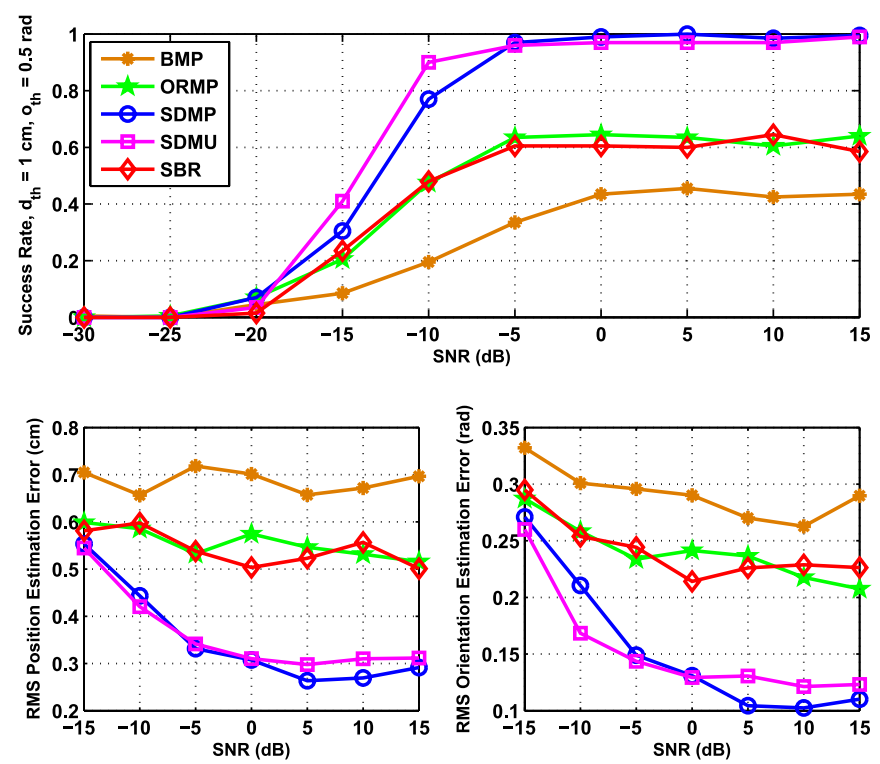

Fig. 4. Performance versus SNR for $95 \%$ correlated signals. Top: success rate, bottom left: RMS position estimation error, bottom right: RMS orientation estimation error as a function of SNR with source separation distance $=7.5 \mathrm{~cm}$, $d_{\mathrm{th}}=1 \mathrm{~cm}$ and $o_{\mathrm{th}}=0.5 \mathrm{rad}$.

the most robust. The ability of SD to reduce the multisource localization problem to a series of single-source problems allows the structured MUSIC-based approach to outperform methods based on unstructured ML.

\section{AUDITORY EXPERIMENTS}

Experiments with auditory stimuli were conducted with a single human subject to elicit auditory-evoked potentials (AEPs). A $1-\mathrm{kHz}$ pure tone audio stimulus of duration $100 \mathrm{~ms}$ was presented to the subject's left ear. The subject was not blindfolded. EEG signals were measured using two NeXus-32 (MindMedia, Roermond-Herten, The Netherlands) systems with linked trigger ports and driven by a synchronization signal so that they were effectively acting as one system. The outputs of 61 electrodes were recorded, similar to the setup assumed in the simulations of the previous section. Normally, auditory stimuli presented at one of the ears are conveyed through auditory pathways until they reach the brain. At the beginning, the inputs cross to the opposite side in the hindbrain and then there is a recrossing of information so that information from the ear reaches both hemispheres [41], [42]. Due to the mechanism described earlier, we might expect to see activity at both primary auditory cortices when the tone is applied to one of the ears of the subject. AEPs associated with the P50 and N100 waves have been reported to be the most relevant this type of auditory experiment; they have latencies of about 50 and $100 \mathrm{~ms}$ from stimulus onset and have a strong contribution from the primary auditory cortex [20], [41]. For our study, the data sample corresponding to the peak of N100 (at about $120 \mathrm{~ms}$ after application of the stimulus in this dataset) was averaged over 288 trials and used to obtain AEP activity for algorithm verification. Further details of the experimental setup and data preprocessing approaches are discussed in [40]. Before 

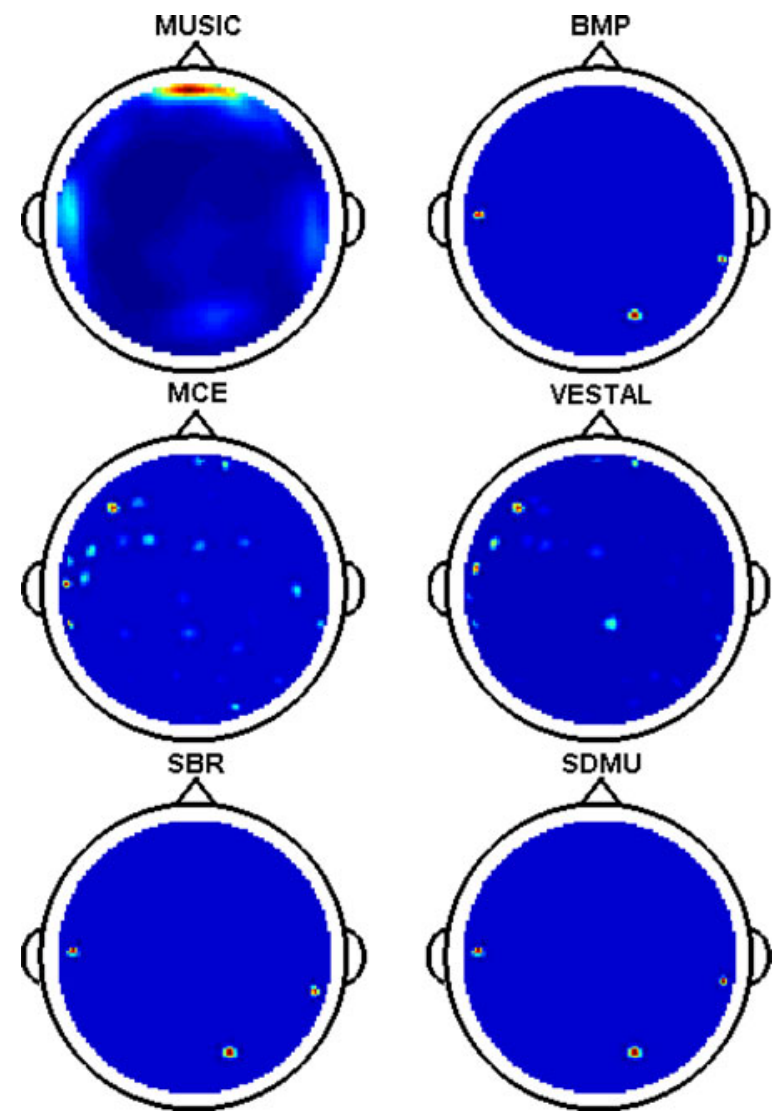

Fig. 5. Estimated source activity obtained by MUSIC, $l_{1}$-norm-based approaches, SBR, and discussed algorithms using experimental activity data. The number of sources was set at 3 .

applying the algorithms, the null-projection technique of [21] was applied to the data for interference suppression.

Fig. 5 shows the estimated source activity obtained by MUSIC, BMP, MCE, VESTAL, SBR, and SDMUSIC for one of the EEG datasets. ORMP gave results similar to BMP, and SDMP gave results similar to SDMUSIC, so their location estimates are not plotted here. Fig. 5 corresponds to the case where the algorithms were told that three sources were present. SBR, $\mathrm{BMP}$, and the proposed SD algorithms provide location estimates that are consistent with the expected locations of cortical activity in response to auditory stimuli. An additional source is localized in the occipital lobe by these three algorithms as well. While the MUSIC algorithm shows activity for the left auditory cortex, its spectrum is smeared and appears to show artifacts that are more likely due to interference from background brain activity than the auditory evoked response. MCE and VESTAL require no knowledge about the number of sources, and in this example, both algorithms provide location estimates around the auditory cortices, although apparent spurious activity shows up as well. This example illustrates the basic difference between sparse methods and classical spectrum-based methods such as MUSIC. The sparse methods directly provide a small number of discrete locations that are the most likely sources of relevant brain activity, while classical methods such as MUSIC provide a "continuous" spectrum that must be searched for peaks, and that has a higher likelihood of false detections.

\section{CONCLUSION}

We have presented SDMP algorithms for EEG/MEG dipole location and moment estimation. We have focused on a model assuming time-invariant dipole orientations (the structured dipole model) and MMVs, although the unstructured case was addressed as well. The advantages of the proposed SDMP approach are that, unlike other MP-based methods for sparse signal models, it eliminates location errors due to residual interference, and, unlike AP-based methods, it provides for an efficient and accurate way of estimating the dipole orientation. A number of simulations were conducted to illustrate the performance advantage of SDMP for various SNR, source separation, and source correlation conditions. Results obtained using real EEG data collected with auditory stimuli also support the validity of the algorithms.

\section{REFERENCES}

[1] J. W. Phillips, R. M. Leahy, and J. C. Mosher, "MEG-based imaging of focal neuronal current sources," IEEE Trans. Med. Imag., vol. 16, no. 3, pp. 338-348, Jun. 1997.

[2] K. Matsuura and Y. Okabe, "Selective minimum-norm solution of the biomagnetic inverse problem," IEEE Trans. Biomed. Eng., vol. 42, no. 6, pp. 608-615, Jun. 1995.

[3] K. Matsuura and Y. Okabe, "A robust reconstruction of sparse biomagnetic sources,” IEEE Trans. Biomed. Eng., vol. 44, no. 8, pp. 720-726, Aug. 1997

[4] I. F. Gorodnitsky, J. S. George, and B. D. Rao, "Neuromagnetic source imaging with FOCUSS: A recursive weighted minimum norm algorithm," J. Electroenceph. Clin. Neurophysiol., vol. 95, pp. 231-251, 1995.

[5] B. D. Rao, "Signal processing with the sparseness constraint," in Proc IEEE Int. Conf. Acoust., Speech, Signal Process, May 1998, vol. 3, pp. 1861-1864.

[6] K. Uutela, M. Hamalainen, and E. Somersalo, "Visualization of magnetoencephalographic data using minimum current estimates," Neuroimage, vol. 10, pp. 173-180, 1999.

[7] M. X. Huang, A. M. Dale, T. Song, E. Halgren, D. L. Harrington, I. Podgorny, J. M. Canive, S. Lewis, and R. R. Lee, "Vector-based spatialtemporal minimum 11-norm solution for MEG," Neuroimage, vol. 31, pp. 1025-1037, 2006.

[8] L. Ding and B. He, "Sparse source imaging in EEG," in Proc. Noninvasive Functional Source Imag. Brain, Heart-Int. Conf. Functional Biomed. Imag., Oct. 2007, pp. 20-23.

[9] W. Ou, M. S. Hamalainen, and P. Golland, "A distributed spatio-tempora EEG/MEG inverse solver,” Neuroimage, vol. 44, pp. 932-946, 2009.

[10] S. C. Wu and A. L. Swindlehurst, "EEG/MEG source localization using source deflated matching pursuit," in Proc. IEEE Eng. Med. Biol. Soc., Aug./Sep. 2011, pp. 6572-6575.

[11] A. Bolstad, B. V. Veen, and R. Nowak, "Space-time event sparse penalization for magneto-electroencephalography," Neuroimage, vol. 46, pp. 1066-1081, 2009.

[12] S. S. Dalal, A. G. Guggisberg, E. Edwards, K. Sekihara, A. M. Findlay, R. T. Canolty, M. S. Berger, R. T. Knight, N. M. Barbaro, H. E. Kirsch, and S. S. Nagarajan, "Five-dimensional neuroimaging: Localization of the time-frequency dynamics of cortical activity," Neuroimage, vol. 40, pp. 1686-1700, 2008.

[13] B. K. Natarajan, "Sparse approximate solutions to linear systems," SIAM J. Comput., vol. 24, pp. 227-234, 1995.

[14] B. D. Rao, K. Engan, S. F. Cotter, J. Palmer, and K. Kreutz-Delgado, "Subset selection in noise based on diversity measure minimization," IEEE Trans. Signal Process., vol. 51, no. 3, pp. 760-769, Mar. 2003.

[15] S. F. Cotter, J. Adler, B. D. Rao, and K. Kreutz-Delgado, "Forward sequential algorithms for best basis selection," Proc. IEE Vis. Imag. Signal Process., vol. 146, pp. 235-244, 1999. 
[16] S. F. Cotter, B. D. Rao, K. Engan, and K. Kreutz-Delgado, "Sparse solutions to linear inverse problems with multiple measurement vectors," IEEE Trans. Signal Process., vol. 53, no. 7, pp. 2477-2488, Jul. 2005.

[17] J. C. Mosher, P. S. Lewis, and R. M. Leahy, "Multiple dipole modeling and localization from spatio-temporal MEG data," IEEE Trans. Biomed. Eng., vol. 39, no. 6, pp. 541-557, Jun. 1992.

[18] J. C. Mosher and R. M. Leahy, "Source localization using recursively applied and projected RAP MUSIC," IEEE Trans. Signal Process., vol. 47, no. 2, pp. 332-340, Feb. 1999.

[19] Z. Zhang and B. Rao, "Sparse signal recovery in the presence of correlated multiple measurement vectors," in Proc. IEEE Int. Conf. Acoust., Speech, Signal Process., Mar. 2010, pp. 3986-3989.

[20] K. Sekihara, K. Hild, and S. Nagarajan, "A novel adaptive beamformer for MEG source reconstruction effective when large background brain activities exist," IEEE Trans. Biomed. Eng., vol. 53, no. 9, pp. 1755-1764, Sep. 2006

[21] S. C. Wu, A. L. Swindlehurst, P. T. Wang, and Z. Nenadic, "Projection versus prewhitening for EEG interference suppression," IEEE Trans. Biomed. Eng., vol. 59, no. 5, pp. 1329-1338, May 2012.

[22] D. Needell and J. A. Tropp, "CoSaMP: Iterative signal recovery from incomplete and inaccurate samples," Appl.Comput. Harmon. Anal., vol. 26 , no. 1, pp. 301-321, 2009.

[23] D. L. Donoho, A. Maleki, and A. Montanari, "Message-passing algorithms for compressed sensing," Proc. Nat. Acad. Sci., vol. 106, pp. 18914 $18919,2009$.

[24] C. Soussen, J. Idier, D. Brie, and J. Duan, "From bernoulli-gaussian deconvolution to sparse signal restoration," IEEE Trans. Signal Process., vol. 59, no. 10, pp. 4572-4584, Oct. 2011.

[25] I. Ziskind and M. Wax, "Maximum likelihood localization of multiple sources by alternating projection," IEEE Trans. Acoust. Speech Signal Process., vol. 36, no. 10, pp. 1553-1560, Oct. 1988.

[26] I. Ziskind and M. Wax, "Optimum localization of coherent signals having diverse polarizations," in Proc. Int. Symp. Antennas Propagat. Soc., 1988 , vol. 1, pp. 250-253.

[27] H. Mohseni and S. Sanei, "A new beamforming-based MEG dipole source localization method," in Proc. IEEE Int. Conf. Acoust., Speech, Signal Process., Mar. 2010, pp. 558-561.

[28] R. Schmidt, "Multiple emitter location and signal parameter estimation," IEEE Trans. Antennas Propag., vol. 34, no. 3, pp. 276-280, Mar. 1986.

[29] J. Sarvas, "Basic mathematical and electromagnetic concepts of the biomagnetic inverse problem," Phys. Med. Biol., vol. 32, pp. 11-22, 1987.

[30] M. Sun, "An efficient algorithm for computing multi-shell spherical volume conductor models in EEG dipole source localization," IEEE Trans. Biomed. Eng., vol. 44, no. 12, pp. 1243-1252, Dec. 1997.

[31] B. N. Cuffin, "EEG localization accuracy improvements using realistically shaped head models," IEEE Trans. Biomed. Eng., vol. 43, no. 3, pp. 299303, Mar. 1996

[32] J. A. Tropp, "Greed is good: Algorithmic results for sparse approximation," IEEE Trans. Inf. Theor., vol. 50, no. 10, pp. 2231-2242, Oct. 2004.

[33] S. K. Oh and C. K. Un, "A sequential estimation approach for performance improvement of eigenstructure-based methods in array processing," IEEE Trans. Signal Process., vol. 41, no. 1, pp. 457-463, Jan. 1993.

[34] B. Lütkenhöner, "Dipole source localization by means of maximum likelihood estimation: Theory and simulations," Electroencephalogr. Clin. Neurophysiol., vol. 106, pp. 314-321, 1998.

[35] B. D. van Veen, W. van Drongelen, M. Yuchtman, and A. Suzuki, "Localization of brain electrical activity via linearly constrained minimum variance spatial filtering," IEEE Trans. Biomed. Eng., vol. 44, no. 9, pp. 867-880, Sep. 1997.

[36] R. Oostenveld and P. Praamstra, "The five percent electrode system for high-resolution EEG and ERP measurements," Clin. Neurophysiol., vol. 112, pp. 713-719, 2001.

[37] B. N. Cuffin and D. Cohen, "Comparison of the magnetoencephalogram and electroencephalogram," Electroencephalogr. Clin. Neurophysiol., vol. 47, pp. 132-146, 1979.

[38] S. J. Luck, An Introduction to the Event-Related Potential Technique. Cambridge, MA, USA: MIT Press, 2005.

[39] K. Sekihara, S. S. Nagarajan, D. Poeppel, and A. Marantz, "Performance of an MEG adaptive-beamformer technique in the presence of correlated neural activities: Correlation-coefficient estimation and time-course retrieval," IEEE Trans. Biomed. Eng., vol. 49, no. 12, pp. 1534-1546, Dec. 2002.
[40] S. C. Wu, A. L. Swindlehurst, P. T. Wang, and Z. Nenadic, "Efficient dipole parameter estimation in EEG systems with near-ML performance," IEEE Trans. Biomed. Eng., vol. 59, no. 5, pp. 1339-1348, May 2012.

[41] E. Pekkonen, M. Huotilainen, J. Virtanen, J. Sinkkonen, T. Rinne, R. J. Ilmoniemi, and R. Näätänen, "Age-related functional differences between auditory cortices: A whole-head MEG study," Neuroreport, vol. 6 , pp. 1803-1806, 1995

[42] B. Kolb and I. Q. Whishaw, Introduction to Brain and Behavior. London, U.K.: Worth, 2001.

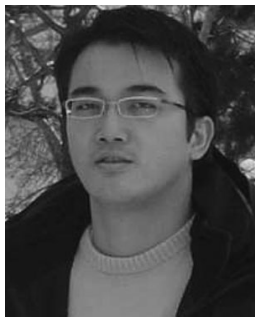

Shun Chi Wu (S'09-M'13) received the B.S. and M.S. degrees in engineering and system science from National Tsing Hua University, Hsinchu, Taiwan, in 2000 and 2002, respectively, and the Ph.D. degree in electrical engineering from the University of California at Irvine, Irvine, CA, USA, in 2012.

From 2003 to 2007, he was a Research Assistant with the National Space Organization, Hsinchu. His research interests include interference mitigation, feature extraction, source localization/reconstruction, and brain connectivity analysis.

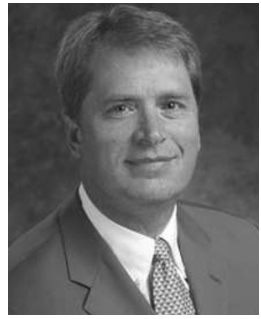

A. Lee Swindlehurst (S'83-M'84-SM'99-F'04) received the B.S. (summa cum laude) and M.S. degrees in electrical engineering from Brigham Young University, Provo, UT, USA, in 1985 and 1986, respectively, and the Ph.D. degree in electrical engineering from Stanford University, Stanford, CA, USA, in 1991.

From 1986 to 1990 , he was with ESL Inc., Sunnyvale, CA, where he was involved in the design of algorithms and architectures for several radar and sonar signal processing systems. He was with the Department of Electrical and Computer Engineering, Brigham Young University, from 1990 to 2007, where he was a Full Professor and served as the Department Chair from 2003 to 2006. During 1996-1997, he was a visiting scholar with both Uppsala University, Uppsala, Sweden, and the Royal Institute of Technology, Stockholm, Sweden. From 2006 to 2007, he was on leave working as a Vice President of Research for ArrayComm LLC, San Jose, CA. He is currently a Professor of electrical engineering and computerscience with the University of California, Irvine, CA. His research interests include sensor array signal processing for radar and wireless communications, detection and estimation theory, and system identification, and he has more than 220 publications in these areas.

Dr. Swindlehurst is a past Secretary of the IEEE Signal Processing Society, past Editor-in-Chief of the IEEE Journal of SELECTED TOPICS IN SIGNAL PROCESSING, and a past member of the Editorial Boards for the EURASIP Journal on Wireless Communications and Networking, IEEE SIGNAL PROCESSING Magazine, and the IEEE Transactions on Signal Processing. He is a recipient of several paper awards: the 2000 IEEE W. R. G. Baker Prize Paper Award, the 2006 and 2010 IEEE Signal Processing Society's Best Paper Award, the 2006 IEEE Communications Society Stephen O. Rice Prize in the Field of Communication Theory, and is a coauthor of a paper that received the IEEE Signal Processing Society Young Author Best Paper Award in 2001. 